This document is confidential and is proprietary to the American Chemical Society and its authors. Do not copy or disclose without written permission. If you have received this item in error, notify the sender and delete all copies.

\title{
Full Characterization of Vibrational Coherence in a Porphyrin Chromophore by Two-Dimensional Electronic Spectroscopy
}

\begin{tabular}{|r|l|}
\hline Journal: & The Journal of Physical Chemistry \\
\hline Manuscript ID: & jp-2014-11881a \\
\hline Manuscript Type: & Article \\
\hline Date Submitted by the Author: & $28-N o v-2014$ \\
\hline Complete List of Authors: & $\begin{array}{l}\text { Camargo, Franco; University of East Anglia, Chemistry } \\
\text { Anderson, Harry; Oxford University, Department of Chemistry } \\
\text { Meech, Stephen; University of East Anglia, Chemical Sciences and } \\
\text { Pharmacy } \\
\text { Heisler, Ismael; University Of East Anglia, Chemistry }\end{array}$ \\
\hline
\end{tabular}


Full Characterization of Vibrational Coherence in a Porphyrin Chromophore by TwoDimensional Electronic Spectroscopy

\author{
Franco V. A. Camargo ${ }^{1}$, Harry L. Anderson ${ }^{2}$, Stephen R. Meech ${ }^{1}$ and Ismael A. Heisler ${ }^{1 *}$ \\ ${ }^{1}$ School of Chemistry, Norwich Research Park, University of East Anglia, Norwich NR4 7TJ, \\ United Kingdom \\ ${ }^{2}$ Department of Chemistry, University of Oxford, Chemistry Research Laboratory, Oxford \\ OX1 3TA, United Kingdom
}

*Corresponding Author: i.heisler@uea.ac.uk 


\begin{abstract}
In this work we present experimental and calculated two-dimensional electronic spectra for a 5,15bisalkynyl porphyrin chromophore. The lowest energy electronic $Q_{y}$ transition couples mainly to a single $380 \mathrm{~cm}^{-1}$ vibrational mode. The two-dimensional electronic spectra reveal diagonal and cross peaks which oscillate as a function of population time. We analyse both the amplitude and phase distribution of this main vibronic transition as a function of excitation and detection frequencies. Even though Feynman diagrams provide a good indication of where the amplitude of the oscillating components are located in the excitation-detection plane, other factors also affect this distribution. Specifically, the oscillation corresponding to each Feynman diagram is expected to have a phase that is a function of excitation and detection frequencies. Therefore, the overall phase of the experimentally observed oscillation will reflect this phase dependence. Another consequence is that the overall oscillation amplitude can show interference patterns resulting from overlapping contributions from neighbouring Feynman diagrams. These observations are consistently reproduced through simulations based on third order perturbation theory coupled to a spectral density described by a Brownian oscillator model.
\end{abstract}

KEYWORDS: ultrafast, 2D electronic spectroscopy, porphyrin, vibrational dynamics 


\section{Introduction}

In order to fully understand the electronic spectra of molecular structures it is essential to take into account the different possible couplings of electronic transitions to intramolecular vibrational modes or, when the molecule is part of an aggregate, to other electronic transitions. ${ }^{1-2}$ With the advent of reliable and stable femtosecond laser systems ultrafast time resolved techniques have been developed to unravel such information. Among those techniques, a powerful method known as two-dimensional electronic spectroscopy (2D ES) has been developed to give direct and precise information about couplings in molecular electronic transitions and at the same time enabling determination of line broadening mechanisms. ${ }^{3}$ The method has been applied to molecular chromophores with different degrees of complexity such as, for instance, dye molecules, nanostructures, molecular aggregates and, above all, light-harvesting complexes. ${ }^{4-11}$

Similarly to pump-probe measurements, 2D spectra contain information about coherent wavepacket evolution during electronic population relaxation time. Such oscillatory dynamics can have a purely vibrational origin, as is the case for independently dissolved chromophores, or an electronic origin, in case of coupled molecular systems like dimers or aggregates. ${ }^{12}$ However, the latter case is difficult to achieve in isolation and in general, electronically coupled systems present a combination of both electronic and vibrational contributions known as vibronic coherence ${ }^{13} \mathrm{~A}$ persistent issue in the field remains the establishment of reliable criteria to assign the vibrational or electronic origin of coherent oscillations observed during population relaxation time. ${ }^{8,12,14}$ This became a critical matter when a number of $2 \mathrm{D}$ ES measurements, performed mainly on the FMO protein complex, presented oscillations with long dephasing times (in the region of ps) during the electronic population relaxation time. ${ }^{15}$ Those oscillations were assigned to electronic coherences, an unexpected result given that electronic coherences typically dephase in tens of femtoseconds. This result opened the possibility that electronic energy could be flowing coherently in light harvesting systems. ${ }^{15-16}$ However, more recent experimental and theoretical work has questioned 
the assignment to pure electronic coherence. ${ }^{17-19}$ One criterion for distinguishing electronic from vibrational coherences, initially proposed by Cheng and Fleming, is the separate analysis of rephasing and nonrephasing $2 \mathrm{D}$ signals. ${ }^{20}$ They showed that for a pure electronic coherence, oscillations will only appear on the diagonal peaks in nonrephasing signals whereas for a rephasing signal oscillations only appear on cross peaks. However, for vibrational coherences oscillations can be present for both nonrephasing and rephasing signals and for any peak position. Even though experimental corroboration was obtained for this criterion the majority of real systems do not present pure vibrational or electronic coherences. ${ }^{12}$ Therefore, another parameter was proposed as a criterion for this assignment: the phase of the oscillations as a function of the excitation and detection frequencies. ${ }^{8,21}$ For example, based on a theoretical analysis it was argued that a $90^{\circ}$ phase shift measured between oscillations on the diagonal and the cross peak in the FMO complex provided evidence of quantum transport effects and therefore electronic coherence. ${ }^{22}$ Another study using a much simpler isolated chromophore showed that indeed phase shifts between the different peaks on the $2 \mathrm{D}$ map are expected, although only $0^{\circ}$ or $180^{\circ}$ degrees phase shifts were forecast and experimentally observed. ${ }^{23}$ This assignment method relies on a clear determination of the absorption peak maxima related to the $0-0$ transition and the first vibronic transition, $0-1$, which is rarely found in solution. A recent theoretical paper proposed that another way to differentiate vibrational from electronic coherences is to measure the relative phase (between diagonal and cross-peaks) as a function of temperature. ${ }^{24}$

Recently a theoretical paper showed explicitly the origin of the phase dependence, as a function of excitation and detection frequencies, for a given Feynman diagram. ${ }^{25}$ Apart from the previously discussed $0^{\circ}$ or $180^{\circ}$ degrees phase shifts observed at the maxima of the peaks on the diagonal and antidiagonal, a further phase dependence is present for frequencies detuned from the absorption maxima. The picture is further complicated by the fact that usually the absorption peak of the main electronic transition and the vibronic progression peak are overlapped. Only for systems with 
electronic transitions coupled to high frequency vibrational modes or with large Huang-Rhys factors can the absorption maxima of the fundamental and first vibronic overtone be clearly identified. Therefore it is becoming apparent that the phase of the oscillations might not be a reliable determinant in assigning coherences either to vibrational or electronic origin.

Many of these conclusions originate from theoretical analysis and therefore experimental verification of the relationship between amplitude and phase in 2D ES is essential. To be able to determine precisely the phase of oscillations as a function of detection and excitation frequencies, 2D ES spectra with good signal/noise are required. In addition, the collection of many 2D ES spectra is necessary to have good sampling of the population time, enabling acquisition of oscillations over a broad frequency range. Due to stringent 2D ES stability requirements, the collection of many 2D ES spectra has to be performed at a high acquisition rate. Our recently developed $2 \mathrm{D}$ ES setup ${ }^{26}$ fulfils such criteria and therefore we performed a comprehensive study of oscillatory dynamics of the $Q_{y}$ band of a porphyrin chromophore. This electronic transition is coupled mainly to a single vibrational mode and therefore serves as an almost ideal four level test system for the study of phase and amplitude distribution of oscillations during population time.

\section{Experimental Methods}

The detailed description of our 2D experimental setup has been presented before. ${ }^{26}$ Briefly, the light source was a commercial amplified laser system plus a noncollinear parametric amplifier (NOPA). The pulses were recompressed close to the Fourier transform limit at the sample position and were characterized by transient grating frequency resolved optical gating (TG-FROG) using a $1 \mathrm{~mm}$ fused silica window. The TG-FROG trace is presented in Figure S1.

The $2 \mathrm{D}$ setup is based on a scheme using two beamsplitters to generate the four phase coherent beams located on the corners of a $2.5 \mathrm{~cm}$ square. During the measurements, the coherence time was scanned in the range from $-150 \mathrm{fs}$ to $150 \mathrm{fs}$ in steps of $4 \mathrm{fs}$. The population time was varied from 
$30 \mathrm{fs}$ up to $1000 \mathrm{fs}$ with $10 \mathrm{fs}$ and after that with increasingly longer steps up to $600 \mathrm{ps}$. The complete data acquisition took around 2 hours.

We also carried out simulations in a similar fashion to previous work..$^{8-9}$ We assumed an electronic two level system and incorporated the nuclear degrees of freedom into the spectral density. Following standard nonlinear response function theory, the spectral density was used as an input to calculate the lineshape function. ${ }^{27}$ This was in-turn used to calculate the nonlinear response functions, linear absorption spectrum and 2D spectra. Only the $Q_{y}$ electronic transition coupled to one underdamped $380 \mathrm{~cm}^{-1}$ vibrational mode was included in the calculation and therefore the linear absorption spectrum fits well up to $16000 \mathrm{~cm}^{-1}$ (see Figure S6a). This is the spectral region of interest in this work and is well captured by the 2D ES measurements. At higher energies additional electronic states contribute to the absorption spectrum. It is also interesting, though not directly relevant, to note that our calculations captured well the spectral diffusion as quantified by the time dependence of the centre line slope (CLS) (see Figure S6b). Unfortunately the oscillations are difficult to reproduce due to numerical calculation artefacts after the multiple Fourier transforms. However the multiexponential relaxation matches the experimental data exactly. Further details and results are presented in supporting information.

The porphyrin chromophore structure is shown in Figure 1 (inset) and was synthesised as reported previously. ${ }^{28}$ It was dissolved in toluene with a concentration of $80 \mu \mathrm{M}$ for a $1 \mathrm{~mm}$ pathlength static cell producing an $\mathrm{OD}$ around 0.3 . Measurements were also done by circulating the solution in a flow cell system, which had a pathlength of $200 \mu \mathrm{m}$ (concentration $400 \mu \mathrm{M}$ ). The results were identical to those in the $1 \mathrm{~mm}$ pathlength cell. The advantage of working with smaller pathlengths is the suppression of contribution from the solvent, but greater dilution in longer pathlength cells reduces the possibility of aggregation; no evidence of aggregation was observed even for the highest concentrations.

\section{Results and Discussion}


The porphyrin chromophore used in this work consists of a bis-meso-ethynyl-substituted zincporphyrin chromophore whose molecular structure is shown as an inset in Figure 1 together with the linear absorption spectrum focusing on the lowest energy $Q$ band. ${ }^{29-30}$ The chromophore consists of a zinc porphyrin ring with attached substituents necessary for high solubility of the molecule in nonpolar solvents. The substituents lower the molecular symmetry from $D_{4 h}$ to $D_{2 h}$ resulting in the splitting of the $Q$ band into two in plane orthogonal transition dipole moments, the $Q_{x}$ and $Q_{y}$ absorption bands. The $\mathrm{Q}_{\mathrm{y}}$ absorption is centred at $15540 \mathrm{~cm}^{-1}$ whereas the $\mathrm{Q}_{\mathrm{x}}$ transition is blue shifted at $16667 \mathrm{~cm}^{-1}$. The band around $16949 \mathrm{~cm}^{-1}$ contains contributions from the $Q_{x}$ transition as well as higher frequency vibronic transitions. The shoulder on the blue side of the $Q_{y}$ transition corresponds to a vibronic transition. The wavenumber of the coupled vibrational mode is around $380 \mathrm{~cm}^{-1}$ and corresponds to the metal-pyrrole breathing mode ( $v_{8}$ mode in usual nomenclature). ${ }^{31}$

The $2 \mathrm{D}$ spectrum for a population time equal to $120 \mathrm{fs}$ is shown in Figure 2 . This spectrum corresponds to the real part of the sum of rephasing and nonrephasing contributions, the absorptive signal. ${ }^{6}$ The top and right graphs also show the laser and linear absorption spectra, which are useful to identify where signal amplitude can be expected as well as identifying the diagonal peaks in the 2D spectrum. The main peak along the diagonal corresponds to the $Q_{y}$ transition (circle $C$ ) centred at $15540 \mathrm{~cm}^{-1}$. Due to a broad higher excited state transition centred at around $30000 \mathrm{~cm}^{-1}$, the vibronic shoulder centred at $15920 \mathrm{~cm}^{-1}$ (circle B) is partly obscured due to overlap with a negative signal originating in excited state absorption. This excited state absorption originates from $S_{1}$ to $S_{3}$ transitions and a fuller characterisation and explanation for it is provided in Figure S4. In fact due to this broad high-energy band a negative signal is expected to cover the entire absorptive 2D mapshowever, only on the wings of the spectrum does it outweigh the positive contributions arising from bleaching and stimulated emission. The 2D map's amplitude axis is shown on a logarithmic scale in order to highlight the low amplitude cross peaks, therefore in Figure 2 all negative features only 
appear as the darkest shade of blue. This is not a limiting choice of scale though, as analysis of regions with overall negative amplitude showed no interesting topography.

The positions indicated by the circles $A, D, E$ and $F$ correspond to spectral regions where oscillations corresponding to a $380 \mathrm{~cm}^{-1}$ mode are expected..$^{9}$ Information about those regions can be obtained by drawing Feynman diagrams which track the time ordered electric field-matter interactions. The Feynman diagrams for the rephasing and nonrephasing contributions are listed in supporting information. In general, either two or four Feynman diagrams give rise to each marked position, and the type of contribution of each diagram can be either a non-oscillating population relaxation or an oscillating vibrational coherence contribution. It is important to note that the Feynman diagram approach does not produce the complete picture because a fixed number of vibrational levels has to be initially specified. In case of only one excited vibrational state there are 16 rephasing and 16 nonrephasing Feynman diagrams contributing to the signal. If higher vibrational excited states are involved, more Feynman diagrams can be drawn and therefore more oscillating contributions may contribute.

Different parameters can be monitored as a function of the population time in order to determine the frequency, amplitude and phase of the coherence oscillation. For example, the ratio between the bandwidth of a cut through the main peak along the diagonal over a cut along the antidiagonal has been used. ${ }^{5,}$ Another possibility is to track the position of the maximum of the main absorption peak, which oscillates with the corresponding frequencies of the vibrational modes coupled to the electronic transition. ${ }^{9}$ However, the most complete information about the coherence oscillations is obtained by following the amplitude of all pairs of detection-excitation frequencies. This can be done by stacking together all the measured 2D maps which, in this work, were a series of 97 measurements starting from $30 \mathrm{fs}$ and going up to $1000 \mathrm{fs}$ in $10 \mathrm{fs}$ time-steps. This was done separately for the rephasing, nonrephasing and absorptive contributions. Figures $3 \mathrm{a}$ and $3 \mathrm{~b}$ show 2D spectra for rephasing and nonrephasing contributions, respectively, for a population time of $100 \mathrm{fs}$. 
The population time dependent amplitude for three different spectral positions for each 2D spectrum is shown in Figures $3 c$ and $3 d$. In each case points 2 and 3 are on the diagonal just above and below circle $\mathrm{C}$ in Figure 2 whereas point 1 corresponds to circle $\mathrm{F}$ in Figure 2 . The time traces are shifted vertically and the non-oscillating population relaxation has been removed. Clear oscillations can be observed for all three spectral positions in both rephasing and nonrephasing contributions. Figure S3 in supporting information presents the power spectrum after taking a Fourier transform of rephasing curve 1 in Figure 3c, clearly showing that the main amplitude peaks around $380 \mathrm{~cm}^{-1}$. Other frequencies are also clearly present. However they are minor contributions and can be mostly assigned to solvent vibrational modes, excited through a Raman scattering process. Another interesting feature which is apparent in the time traces shown in Figures $3 c$ and $3 d$ is the phase difference between the different curves. Rephasing curves 2 and 3 are in phase whereas both those curves are out of phase with curve 1. For the nonrephasing curves the opposite behaviour is seen: curves 2 and 3 are out of phase whereas curves 1 and 2 are in phase. This result points to the fact that for rephasing signals oscillations along the diagonal are in phase whereas the opposite is true for nonrephasing signals.

In addition, the oscillation amplitude is different for different spectral positions on the 2D map. A complete picture of the amplitude and phase distribution of the oscillation can be obtained by subtracting the non-oscillating population relaxation for each pair of detection-excitation frequencies and then taking a Fourier transform. This procedure, which was proposed and implemented by other groups, ${ }^{33-35}$ generates a 3D matrix where the first dimension is the detection frequency, the second dimension is the excitation frequency and the third dimension is the Fourier transform frequency. This 3D matrix can be scanned for the amplitude and phase of the oscillating frequency with wavenumbers around $380 \mathrm{~cm}^{-1}$. The collected data are then plotted on a 2D map as a function of detection-excitation frequencies showing the amplitude and phase distribution of the 
$380 \mathrm{~cm}^{-1}$ mode on the 2D map. The amplitude distribution for rephasing and nonrephasing contributions are shown in Figures 3e and 3f, respectively.

The $380 \mathrm{~cm}^{-1}$ mode amplitude distribution shows an interesting pattern over the $2 \mathrm{D}$ map. For the rephasing contribution there is more amplitude located below the diagonal when compared to the nonrephasing contribution. ${ }^{35}$ This agrees well with what would be expected from an initial inspection of the 2D spectra together with the respective Feynman diagrams: there are two extra oscillating contributions located below the diagonal for the rephasing contribution (Feynman diagrams in circle $\mathrm{F}$, see supporting information). However the regions with no amplitude have a well defined pattern parallel to the diagonal for the rephasing contribution and parallel to the antidiagonal for the nonrephasing contribution, indicating interference minima along those directions. This is further supported by the fact that the resulting phase distribution follows the same pattern, as shown in Figures $4 a$ and $4 b$. These figures show the phase distribution as a colour scale 2D graph with the amplitude distribution plotted on top as contour lines. For the rephasing contribution, the constant phase lines are parallel to the diagonal whereas for the nonrephasing they are parallel to the antidiagonal. Significantly, the experimental results show that the phase of the oscillation is a function of the detection and excitation frequencies and varies significantly over the 2D map. This phase dependence was already noted before and its origin critically discussed in terms of vibronic and Herzberg-Teller couplings. ${ }^{36}$ However the molecular structure, given by chlorosomes, was significantly more complex than the porphyrin molecule discussed here, where excitons and other couplings can be discarded. As noted before (Figure 3), for the rephasing signal oscillations along the diagonal remain approximately in phase, whereas along the antidiagonal the phase can vary by as much as $2 \pi$. The opposite is true for the nonrephasing signals. Our experimental results are in agreement with theoretical results of Butkus et al. where it was shown that the phase of the vibrational coherence oscillation is a function of the detection and excitation frequencies. ${ }^{25}$ In their theoretical work the phase was obtained for a single Feynman diagram 
contribution, which cannot be measured experimentally in isolation. Instead, the experimental signal is always a sum of at least two oscillating contributions. Also, in general and certainly in the present case there is a significant spectral overlap between the $0-0$ and $0-1$ transitions and therefore different Feynman diagram contributions will interfere. In order to show that this is not an artefact due to finite laser pulse widths or pulse overlap we performed simulations for a two level system plus an underdamped spectral density accounting for the vibrational mode (details in supporting information). The simulation results, shown in Figures $4 \mathrm{c}$ and $4 \mathrm{~d}$, are in good qualitative agreement with the experimental results. The same phase variation pattern is observed for the simulations and experimental results as well as a similar amplitude distribution for the corresponding frequencies of the oscillation.

Finally, we discuss the origin of the phase variation across the 2D map. By assuming a homogeneous relaxation mechanism, Butkus et al obtained an analytical expression for a given Feynman diagram where the amplitude and the phase dependence on the dephasing parameter appears explicitly. ${ }^{25}$ Due to the finite linewidth, detuning from the main transition introduces a phase shift between oscillations. This conclusion cannot be reached through a diagrammatic analysis alone because there is an implicit assumption that the energy levels are infinitely well defined. ${ }^{23}$ However, experimentally, vibronic transitions are always broadened and therefore excitation and detection will always have some detuning. Our experimental result is a clear example of this effect and suggests that interpretations relying solely on phase variations might not always produce correct results. For complex systems, where the disentanglement of many overlapping contributions is challenging, the amplitude maxima of oscillatory signals in 2D maps might not correspond to expectations based solely on Feynman diagrams. Therefore phase shifts between oscillations associated with different pairs of excitation-detection frequencies is on its own not a reliable criterion with which to establish assignments, unless they are supported by other evidence.

\section{Conclusions}


In summary, we presented and discussed experimental results obtained by $2 \mathrm{D}$ ES for the $\mathrm{Q}_{\mathrm{y}}$ electronic transition of a porphyrin chromophore coupled to a single intramolecular vibrational mode. The coherently excited vibrational wavepacket modulates the electronic population relaxation, producing an oscillating signal. We focused specifically on the oscillating signal during the electronic population time and determined how the amplitude and the phase of these oscillations are distributed over the 2D map. The phase of the oscillation is clearly a function of excitation and detection frequencies. This experimental result corroborates previously obtained theoretical results which showed that for broadened transitions the phase of the coherent oscillation should be dependent of excitation and detection frequencies. ${ }^{25}$ The results presented here thus question the reliability of assignments on coherence to an electronic or vibrational origin solely on the basis of phase shifts between peaks in a 2D spectrum. 
Supporting Information. Available.

Acknowledgements. This work was supported by EPSRC through grant EP/J009148/1 and J021431/1. Franco Valduga de Almeida Camargo is supported by Brazilian Funding Agency CAPES (Coordination for the Improvement of Higher Level Personnel) through a doctoral studentship (process number BEX 9527/13-3). 
Figure 1. Linear absorption spectrum for the porphyrin chromophore dissolved in toluene in the region of $Q_{y}$ band (blue) along with the laser spectrum (red). Inset shows the molecular structure and the arrows show the $\mathrm{Q}_{\mathrm{x}}$ and $\mathrm{Q}_{\mathrm{y}}$ transition dipole moment directions. 
1

2

3

4

5

6

7

8

9

10

11

12

13

14

15

16

17

18

19

20

21

22

23

24

25

26

27

28

29

30

31

32

33

34

35

36

37

38

39

40

41

42

43

44

45

46

47

48

49

50

51

52

53

54

55

56

57

58

59

60

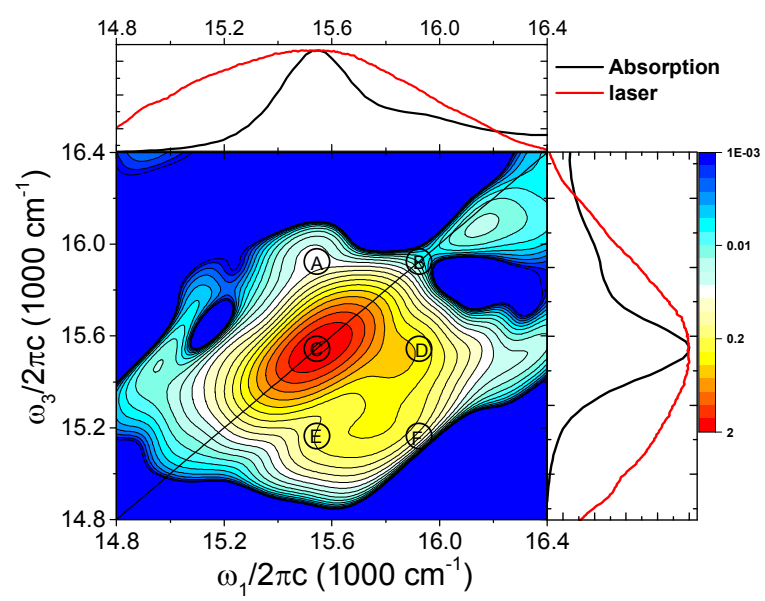

Figure 2. Absorptive 2D ES spectrum for a population time equal to $120 \mathrm{fs}$ (which is a maximum of the oscillation). The top and right side graphs show the linear absorption spectrum (black line) and the laser spectrum (red line). The 2D plot vertical ( $y$ axis) corresponds to detection wavenumbers whereas the horizontal ( $x$ axis) corresponds to the excitation wavenumbers. The amplitude ( $z$ axis) has 21 contour lines evenly spaced on a $\log _{10}$ scale. Circles with letters inside mark positions where Feynman diagrams predict oscillating coherences during the population time. 


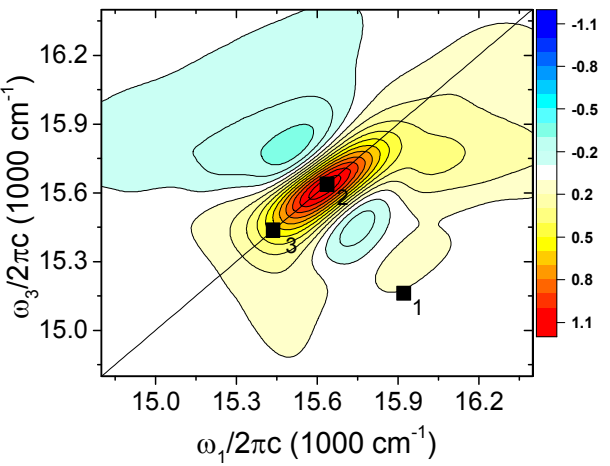

(a)

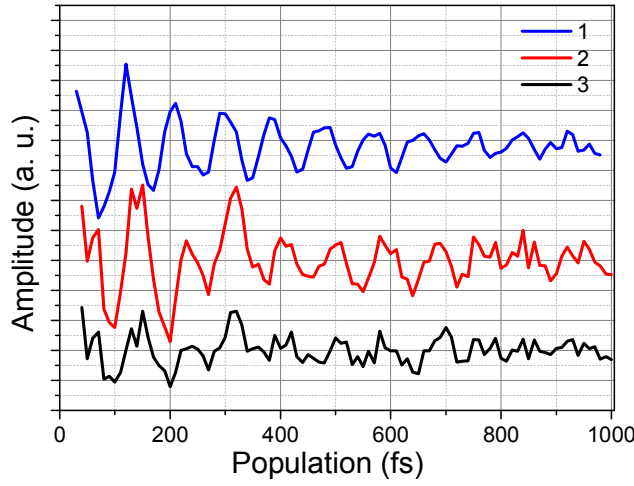

(c)

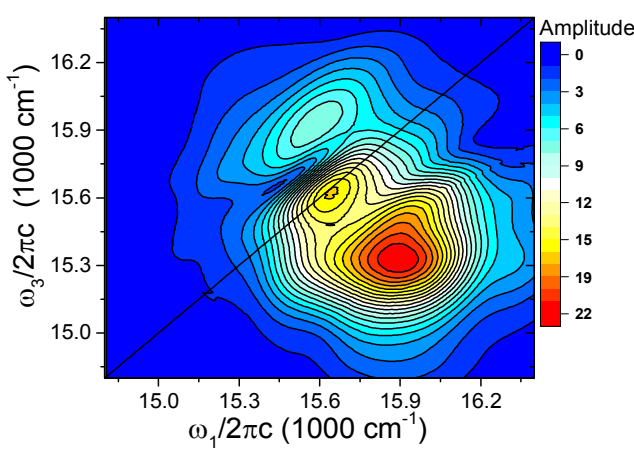

(e)

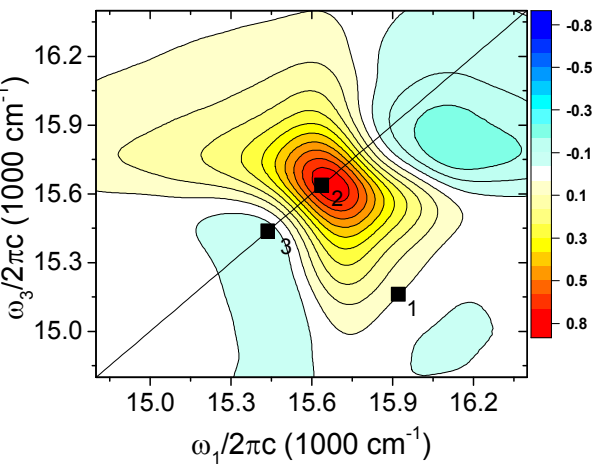

(b)

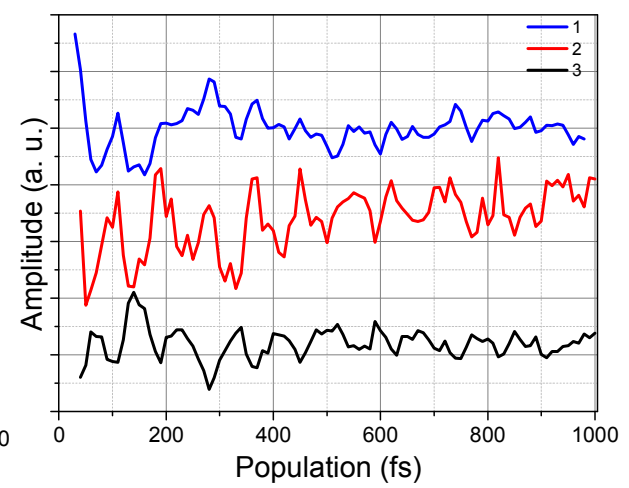

(d)

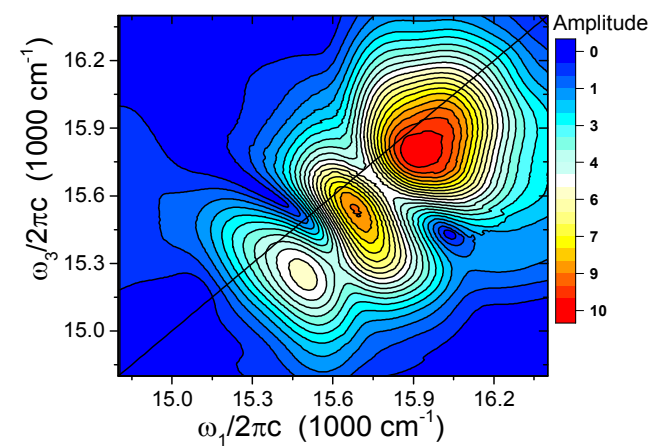

(f)

Figure 3. Graphs (a) and (b) show real rephasing and nonrephasing 2D ES spectra, respectively, for a population time equal to $120 \mathrm{fs}$. The amplitude axis has 21 evenly spaced contour lines on a linear scale. The squares labelled 1 to 3 mark positions where amplitude is followed as a function of population time and shown in graphs (c) and (d) for rephasing and nonrephasing signals, respectively. The curves are offset vertically for better visualization. Graphs (e) and (f) present the 
1

2

3

4

5

6

7

8

9

10

11

12

13

14

15

16

17

18

19

20

21

22

23

24

25

26

27

28

29

30

31

32

33

34

35

36

37

38

39

40

41

42

43

44

45

46

47

48

49

50

51

52

53

54

55

56

57

58

59

60

$380 \mathrm{~cm}^{-1}$ oscillation amplitude distribution as a 2D plot. The amplitude axis shows 21 evenly spaced contour lines on a linear scale. 


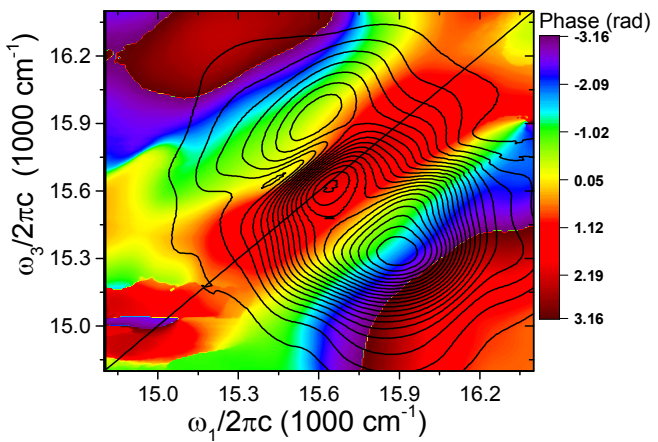

(a)

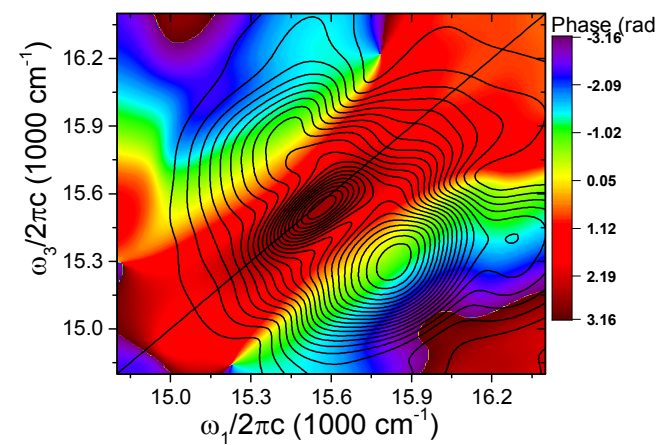

(c)

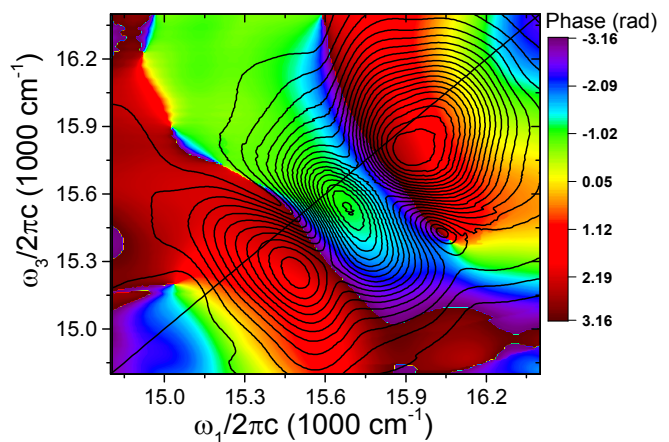

(b)

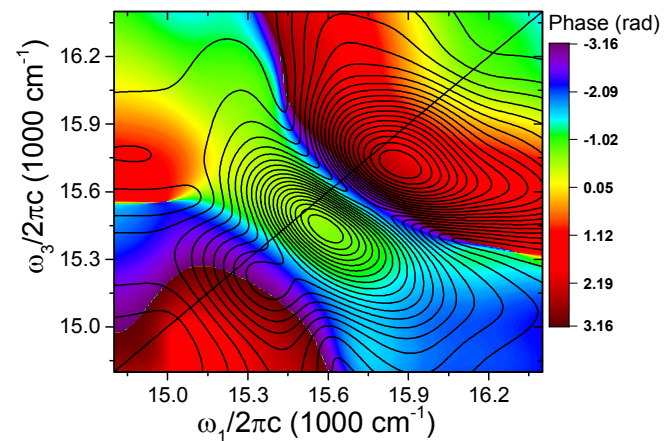

(d)

Figure 4. Graphs (a) and (b) show the distribution of the phase of the $380 \mathrm{~cm}^{-1}$ mode over the 2D map for the measured rephasing and nonrephasing signals, respectively. The phase value is shown as a colour scale (see colour table on the right of the graph). Overlapped is the amplitude distribution (same as Figure $3 a$ and $3 b$ ) shown as contour lines only. The aim is to help identify regions where meaningful signal is expected. Graphs (c) and (d) are same as (a) and (b) but for calculated 2D spectra (see supporting information). 
1. Fleming, G. R.; Cho, M. H., Chromophore-Solvent Dynamics. Annu. Rev. Phys. Chem. 1996, 47, 109-134.

2. de Boeij, W. P.; Pshenichnikov, M. S.; Wiersma, D. A., Ultrafast Solvation Dynamics Explored by Femtosecond Photon Echo Spectroscopies. Annu. Rev. Phys. Chem. 1998, 49, 99-123.

3. Jonas, D. M., Two-Dimensional Femtosecond Spectroscopy. Annu. Rev. Phys. Chem. 2003, 54, 425-463.

4. Cheng, Y. C.; Fleming, G. R., Dynamics of Light Harvesting in Photosynthesis. Annu. Rev. Phys. Chem. 2009, 60, 241-262.

5. Ginsberg, N. S.; Cheng, Y. C.; Fleming, G. R., Two-Dimensional Electronic Spectroscopy of Molecular Aggregates. Accounts Chem. Res. 2009, 42, 1352-1363.

6. Schlau-Cohen, G. S.; Ishizaki, A.; Fleming, G. R., Two-Dimensional Electronic Spectroscopy and Photosynthesis: Fundamentals and Applications to Photosynthetic LightHarvesting. Chem. Phys. 2011, 386, 1-22.

7. Milota, F.; Sperling, J.; Nemeth, A.; Mancal, T.; Kauffmann, H. F., Two-Dimensional Electronic Spectroscopy of Molecular Excitons. Accounts Chem. Res. 2009, 42, 1364-1374.

8. Christensson, N.; Milota, F.; Hauer, J.; Sperling, J.; Bixner, O.; Nemeth, A.; Kauffmann, H. F., High Frequency Vibrational Modulations in Two-Dimensional Electronic Spectra and Their Resemblance to Electronic Coherence Signatures. J. Phys. Chem. B 2011, 115, 5383-5391.

9. Caram, J. R.; Fidler, A. F.; Engel, G. S., Excited and Ground State Vibrational Dynamics Revealed by Two-Dimensional Electronic Spectroscopy. J. Chem. Phys. 2012, 137.

10. Hwang, I.; Selig, U.; Chen, S. S. Y.; Shaw, P. E.; Brixner, T.; Burn, P. L.; Scholes, G. D., Photophysics of Delocalized Excitons in Carbazole Dendrimers. J. Phys. Chem. A 2013, $117,6270-6278$.

11. Dostal, J.; Mancal, T.; Augulis, R.; Vacha, F.; Psencik, J.; Zigmantas, D., TwoDimensional Electronic Spectroscopy Reveals Ultrafast Energy Diffusion in Chlorosomes. $J$. Am. Chem. Soc. 2012, 134, 11611-11617.

12. Turner, D. B.; Wilk, K. E.; Curmi, P. M. G.; Scholes, G. D., Comparison of Electronic and Vibrational Coherence Measured by Two-Dimensional Electronic Spectroscopy. Journal of Physical Chemistry Letters 2011, 2, 1904-1911.

13. Butkus, V.; Valkunas, L.; Abramavicius, D., Vibronic Phenomena and ExcitonVibrational Interference in Two-Dimensional Spectra of Molecular Aggregates. J. Chem. Phys. 2014, 140.

14. Butkus, V.; Zigmantas, D.; Abramavicius, D.; Valkunas, L., Distinctive Character of Electronic and Vibrational Coherences in Disordered Molecular Aggregates. Chem. Phys. Lett. 2013, 587, 93-98.

15. Engel, G. S.; Calhoun, T. R.; Read, E. L.; Ahn, T. K.; Mancal, T.; Cheng, Y. C.; Blankenship, R. E.; Fleming, G. R., Evidence for Wavelike Energy Transfer through Quantum Coherence in Photosynthetic Systems. Nature 2007, 446, 782-786.

16. Panitchayangkoon, G.; Hayes, D.; Fransted, K. A.; Caram, J. R.; Harel, E.; Wen, J. Z.; Blankenship, R. E.; Engel, G. S., Long-Lived Quantum Coherence in Photosynthetic 
Complexes at Physiological Temperature. Proc. Natl. Acad. Sci. U. S. A. 2010, 107, 1276612770.

17. Pullerits, T.; Zigmantas, D.; Sundstrom, V., Beatings in Electronic 2d Spectroscopy Suggest Another Role of Vibrations in Photosynthetic Light Harvesting. Proc. Natl. Acad. Sci. U. S. A. 2013, 110, 1148-1149.

18. Tiwari, V.; Peters, W. K.; Jonas, D. M., Electronic Resonance with Anticorrelated Pigment Vibrations Drives Photosynthetic Energy Transfer Outside the Adiabatic Framework. Proc. Natl. Acad. Sci. U. S. A. 2013, 110, 1203-1208.

19. Chenu, A.; Christensson, N.; Kauffmann, H. F.; Mancal, T., Enhancement of Vibronic and Ground-State Vibrational Coherences in 2d Spectra of Photosynthetic Complexes. Sci Rep-Uk 2013, 3.

20. Cheng, Y. C.; Fleming, G. R., Coherence Quantum Beats in Two-Dimensional Electronic Spectroscopy. J. Phys. Chem. A 2008, 112, 4254-4260.

21. Pisliakov, A. V.; Mancal, T.; Fleming, G. R., Two-Dimensional Optical Three-Pulse Photon Echo Spectroscopy. Ii. Signatures of Coherent Electronic Motion and Exciton Population Transfer in Dimer Two-Dimensional Spectra. J. Chem. Phys. 2006, 124.

22. Panitchayangkoon, G.; Voronine, D. V.; Abramavicius, D.; Caram, J. R.; Lewis, N. H. C.; Mukamel, S.; Engel, G. S., Direct Evidence of Quantum Transport in Photosynthetic Light-Harvesting Complexes. Proc. Natl. Acad. Sci. U. S. A. 2011, 108, 20908-20912.

23. Mancal, T.; Christensson, N.; Lukes, V.; Milota, F.; Bixner, O.; Kauffmann, H. F.; Hauer, J., System-Dependent Signatures of Electronic and Vibrational Coherences in Electronic Two-Dimensional Spectra. Journal of Physical Chemistry Letters 2012, 3, 1497 1502.

24. Perlik, V.; Lincoln, C.; Sanda, F.; Hauer, J., Distinguishing Electronic and Vibronic Coherence in $2 \mathrm{~d}$ Spectra by Their Temperature Dependence. Journal of Physical Chemistry Letters 2014, 5, 404-407.

25. Butkus, V.; Zigmantas, D.; Valkunas, L.; Abramavicius, D., Vibrational Vs. Electronic Coherences in 2d Spectrum of Molecular Systems. Chem. Phys. Lett. 2012, 545, 40-43.

26. Heisler, I. A.; Moca, R.; Camargo, F. V. A.; Meech, S. R., Two-Dimensional Electronic Spectroscopy Based on Conventional Optics and Fast Dual Chopper Data Acquisition. Rev. Sci. Instrum. 2014, 85.

27. deBoeij, W. P.; Pshenichnikov, M. S.; Wiersma, D. A., System-Bath Correlation Function Probed by Conventional and Time-Gated Stimulated Photon Echo. J. Phys. Chem. 1996, 100, 11806-11823.

28. Koszelewski, D., et al., Synthesis and Linear and Nonlinear Optical Properties of Low-Melting Pi-Extended Porphyrins. J Mater Chem C 2013, 1, 2044-2053.

29. Drobizhev, M.; Stepanenko, Y.; Dzenis, Y.; Karotki, A.; Rebane, A.; Taylor, P. N.; Anderson, H. L., Extremely Strong near-Ir Two-Photon Absorption in Conjugated Porphyrin Dimers: Quantitative Description with Three-Essential-States Model. J. Phys. Chem. B 2005, 109, 7223-7236.

30. Anderson, H. L., Conjugated Porphyrin Ladders. Inorg. Chem. 1994, 33, 972-981.

31. Atamian, M.; Donohoe, R. J.; Lindsey, J. S.; Bocian, D. F., Resonance RamanSpectra and Normal-Coordinate Analysis of Reduced Porphyrins .1. Zinc(Ii) Tetraphenylporphyrin Anion. J. Phys. Chem. 1989, 93, 2236-2243.

32. Nemeth, A.; Lukes, V.; Sperling, J.; Milota, F.; Kauffmann, H. F.; Mancal, T., TwoDimensional Electronic Spectra of an Aggregating Dye: Simultaneous Measurement of Monomeric and Dimeric Line-Shapes. Phys. Chem. Chem. Phys. 2009, 11, 5986-5997. 
33. Seibt, J.; Pullerits, T., Beating Signals in 2d Spectroscopy: Electronic or Nuclear Coherences? Application to a Quantum Dot Model System. J. Phys. Chem. C 2013, 117, 18728-18737.

34. Milota, F.; Prokhorenko, V. I.; Mancal, T.; von Berlepsch, H.; Bixner, O.; Kauffmann, H. F.; Hauer, J., Vibronic and Vibrational Coherences in Two-Dimensional Electronic Spectra of Supramolecular J-Aggregates. J. Phys. Chem. A 2013, 117, 6007-6014.

35. Turner, D. B.; Dinshaw, R.; Lee, K. K.; Belsley, M. S.; Wilk, K. E.; Curmi, P. M. G.; Scholes, G. D., Quantitative Investigations of Quantum Coherence for a Light-Harvesting Protein at Conditions Simulating Photosynthesis. Phys. Chem. Chem. Phys. 2012, 14, 48574874.

36. Dostal, J.; Mancal, T.; Vacha, F.; Psencik, J.; Zigmantas, D., Unraveling the Nature of Coherent Beatings in Chlorosomes. J. Chem. Phys. 2014, 140.

\section{TOC GRAPHICS}

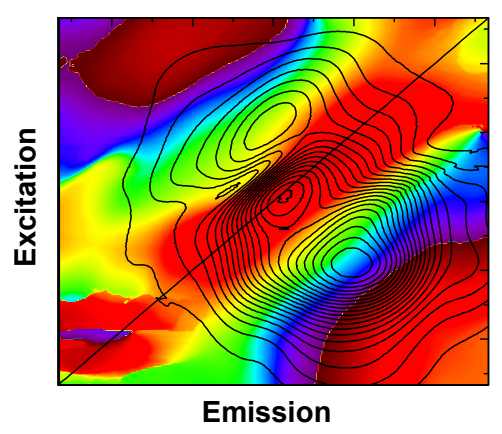

